\title{
Single Crystal Growth and X-ray Structure Analysis of Non-Peripheral Octahexyl Phthalocyanine
}

Masashi Ohmori, Chika Nakano, Tetsuya Miyano, Norimitsu Tohnai, Akihiko Fujii", and Masanori Ozaki

Affiliation: Division of Electrical, Electronic and information Engineering, Graduate School of Engineering, Osaka University

Mailing Address: Suita, Osaka 565-0871, Japan

Phone: +81-6-6879-7758

Fax: $+81-6-6879-4838$

E-mail: afujii@ opal.eei.eng.osaka-u.ac.jp

\begin{abstract}
The single-crystal structure of metal-free non-peripheral octahexyl-substituted phthalocyanine $\left(\mathrm{C} \mathrm{PcH}_{2}\right)$ has been investigated by single-crystal X-ray structure analysis. Two types of $\mathrm{C} \mathrm{PcH}_{2}$ single crystal, bulk and needle crystals, were separately grown by controlling the recrystallization conditions. The structures of the two types of crystal were determined, and were found to be completely different, that is, $\mathrm{C} \mathrm{PcH}_{2}$ exhibits structural polymorphism. It has been clarified that the $\mathrm{C} \mathrm{PcH}_{2}$ microcrystals in thin films used in previously reported electronic devices have the needle structure.
\end{abstract}

Keyword: crystal polymorphism, phthalocyanine, single crystal X-ray structure analysis 


\section{INTRODUCTION}

Phthalocyanines possess a two-dimensional $\pi$-electron conjugated system, and some of them form a columnar structure with a strong one-dimensional $\pi$-orbital overlap existing along the column axis [1]. In the field of organic electronics, phthalocyanines are well known as low-molecular-weight organic semiconductors and have attracted considerable attention for their application to optoelectronic devices, such as organic solar cells $[2,3]$.

The introduction of alkyl substituents into non-peripheral positions of a phthalocyanine core to balance high crystallinity and solubility has been investigated [4-7]. Some non-peripheral alkyl-substituted phthalocyanines have a discotic liquid crystalline phase and self-assemble into columnar stacks [8,9]. Non-peripheral alkyl-substituted phthalocyanines exhibit high carrier mobilities on the order of $10^{-1}$ $\mathrm{cm}^{2} /$ Vs. This is considered to be due to the molecular packing structure.

In particular, the non-peripheral hexyl-substituted phthalocyanine 1,4,8,11,15,18,22,25-octahexyl-phthalocyanine $\left(\mathrm{C} \mathrm{PcH}_{2}\right)$ exhibits an ambipolar high carrier drift mobility exceeding $1 \mathrm{~cm}^{2} / \mathrm{Vs}$ in the crystalline phase, which was evaluated by time-of-flight measurement [10]. Organic thin-film solar cells with an active layer based on a blend film of $\mathrm{C}_{6 \mathrm{PcH}}$ and the fullerene derivative 1-(3-methoxycarbonyl)-propyl-1-phenyl-(6,6)C61 (PCBM) exhibit a high efficiency of $4.2 \%$ [11-13]. Such high mobility and the photovoltaic property are expected to be related to the self-organized molecular packing structure of $\mathrm{C} \mathrm{PcH}_{2}[14,15]$. Therefore, the molecular packing structures of $\mathrm{C} \mathrm{PcH}_{2}$ in the liquid crystal phase and crystal phase have been previously studied [16-19].

In the liquid crystal phase, $\mathrm{C} \mathrm{PcH}_{2}$ forms a pseudo-disordered hexagonal columnar 
mesophase with a column interval of approximately $21 \AA$ as estimated by X-ray diffraction (XRD) measurement [16]. On the other hand, the $\mathrm{C} \mathrm{PcH}_{2}$ single-crystal structure has been classified into the triclinic structure with space group $P-1, a=9.267$ $\AA, b=9.732 \AA, c=19.991 \AA, \alpha=100.10^{\circ}, \beta=91.17^{\circ}, \gamma=95.90^{\circ}$, and $V=1759.2 \AA^{3}$, where $V$ is the volume of the unit cell [19]. Therefore, it is clear that the molecular packing structures in the liquid crystal phase and crystal phase are clearly different.

For device applications, it is necessary to clarify the molecular packing structure of $\mathrm{C}_{6} \mathrm{PcH}_{2}$ in a spin-coated film. This structure was found to be a centered rectangular structure (long side $=36.4 \AA$, short side $=20.3 \AA$ ), and the stacking interval was calculated to be 4.8-5.0 $\AA$ by XRD measurement using a two-dimensional detector [18]. The phthalocyanine core normal vectors were tilted $34-39^{\circ}$ from the column axis. In the case of the spin-coated film, it is difficult to determine the correct structure between two possible molecular packing structures, in which the molecules tilt uniformly or alternately. Figure 1 shows a schematic explanation of the molecular alignment from the direction of the columnar axis and the in-plane direction of the $\mathrm{C} \mathrm{PcH}_{2}$ film. The molecular packing structure in the spin-coated film resembles that of the liquid crystal phase rather than that of the single crystal. This result suggests that crystal polymorphism exists in the crystal phase of $\mathrm{C} \mathrm{PcH}_{2}$.

Since a spin-coated film of $\mathrm{C} \mathrm{PcH}_{2}$ consists of the polycrystal, the crystal structure of $\mathrm{C}_{6 \mathrm{PcH}}$ in the spin-coated film has not been determined in detail and it has been difficult to explain the origin of the high mobility and photovoltaic properties [20,21]. To clarify the detailed crystal structure of $\mathrm{C} \mathrm{PcH}_{2}$ in the spin-coated film, it is important to find a single crystal with a structure similar to the spin-coated film.

In this study, single crystals of $\mathrm{C} \mathrm{PcH}_{2}$ were grown by the recrystallization method 
under various conditions, and the crystal structures were determined by single-crystal X-ray structure analysis. Moreover, the mechanism of the crystal growth was investigated by using the results of the differential scanning calorimetry (DSC) and $\mathrm{X}$-ray structure analysis at various temperature. The molecular packing structure of a spin-coated film is discussed by taking the structures of the single crystals into consideration.

\section{EXPERIMENTAL SECTION}

In this study, the single crystals were fabricated by the following two methods.

Method 1: A saturated $\mathrm{C} \mathrm{PcH}_{2}$ solution was prepared in screw-top vials, and the solvent was vaporized for a month at room temperature under atmospheric conditions. A mixed solvent of chloroform and 2-propanol was used as the recrystallization solvent.

Method 2: A supersaturated $\mathrm{C}_{6} \mathrm{PcH}_{2}$ solution was prepared in screw-top vials at room temperature under atmospheric conditions. The solution was heated at approximately $353 \mathrm{~K}$ to dissolve the $\mathrm{C} \mathrm{PcH}_{2}$ in the solvent and gradually cooled to room temperature over 3 days. A mixture of toluene and acetonitrile was used as the recrystallization solvent.

Diffraction data of the single crystals fabricated by method 1 were collected at $213 \mathrm{~K}$ using a RAPID imaging plate with a two-dimensional area detector and graphite-monochromatized $\mathrm{Cu} \mathrm{K} \alpha$ radiation (RIGAKU R-AXIS RAPID). The crystallographic calculation was performed with a crystal structure analysis package (RIGAKU Crystal Structure 4.0). The diffraction data were also collected at $273 \mathrm{~K}$ using a curved imaging plate (RIGAKU R-AXIS RAPID-191R) with an X-ray generator (RIGAKU FR-E ${ }^{++}$SuperBright) and $\mathrm{Cu} \mathrm{K \alpha}$ radiation. The crystallographic 
calculation was performed by Crystal Structure 4.1. The crystal structure was solved by a direct method (SIR-2008).

Diffraction data of the single crystals fabricated by method 2 were collected at $93 \mathrm{~K}$ using synchrotron radiation $(\lambda=0.8000 \AA)$ at the BL38B1 beamline at SPring- 8 with the approval of JASRI (2015A1776). Cell refinements were performed with HKL2000 software. The crystal structure was solved by a direct method (SIR-2004). The diffraction data has also collected at $273 \mathrm{~K}$ using RIGAKU R-AXIS RAPID. The crystallographic calculation was performed with Crystal Structure 4.1. The crystal structure was solved by a direct method (SHELXS-2013).

The temperature dependences of the lattice constants of the single crystals fabricated by method 2 were measured using RIGAKU FR-E ${ }^{++}$SuperBright.

The thermal phase transition temperature of the single crystal was determined by DSC (TA Instruments Q2000), and the transform of the single crystal structure was observed using an X-ray diffractometer (Rigaku SmartLab) with a $\mathrm{Cu} \mathrm{K} \alpha$ line and heating stage (Anton-Paar DHS900).

\section{RESULTS AND DISCUSSION}

The single crystals fabricated by method 1 had a cuboidal shape, while those fabricated by method 2 had a needle shape, which we refer to as bulk and needle crystals, respectively. Figure 2 shows microscopic images of the bulk and needle crystals. The typical size of the bulk crystals was $1.2 \mathrm{~mm} \times 0.8 \mathrm{~mm}$ and the width of the needle crystals was approximately $100 \mu \mathrm{m}$. Both single crystals were dark and had angular pieces.

The bulk crystal structure at $213 \mathrm{~K}$ was determined to be the triclinic structure with 
space group $P-1, a=9.24911(17) \AA, b=9.70418(18) \AA, c=19.7517(4) \AA, \alpha=$ $100.0230(9)^{\mathbf{o}}, \beta=91.0720(9)^{\mathbf{o}}, \gamma=95.3019(10)^{\mathbf{o}}$, and $V=1737.19 \AA^{3}$. The unit cell contained one molecule and the density was $1.134 \mathrm{~g} / \mathrm{cm}^{3}$. The phthalocyanine core normal vectors were tilted in the same direction. The shortest intermolecular distance was $3.493 \AA$. The tilt angle of the phthalocyanine core normal vectors from the $a$ axis was $67.8^{\circ}$. Figure 3 shows the molecular packing structure of the bulk crystal in the direction of the columnar axis and the in-plane direction at $213 \mathrm{~K}$. This result was consistent with a previously reported result [19].

The molecular packing structure in a spin-coated film at room temperature was reported to be a centered rectangular structure with space group $C 2 / m, a=20.3 \AA, b=$ 4.8-5.0 $\AA, c=36.4 \AA, \alpha=\beta=\gamma=90^{\circ}$, and $V=3.55-3.69 \times 10^{3} \AA^{3}$ [18]. The unit cell contained two molecules and the density was $1.07-1.11 \mathrm{~g} / \mathrm{cm}^{3}$. It is clear that the lattice constants in the spin-coated film are different from those of the bulk crystal at $213 \mathrm{~K}$. To discuss the difference, the structure of the bulk crystal should be analyzed at around room temperature because of the crystalline phase transition at $243 \mathrm{~K}$ in a DSC measurement.

The structure of the bulk crystal was investigated at a higher temperature than the phase transition temperature of $243 \mathrm{~K}$. The bulk crystal structure at $273 \mathrm{~K}$ was determined to be a triclinic structure with space group $P-1, a=9.3149(4) \AA, b=$ $9.7909(5) \AA, c=19.9810(14) \AA, \alpha=100.534(7)^{\circ}, \beta=91.084(6)^{\circ}, \gamma=95.653(7)^{\circ}$, and $V$ $=1781.6 \AA^{3}$. The unit cell contained one molecule and the density was $1.106 \mathrm{~g} / \mathrm{cm}^{3}$. The phthalocyanine core normal vectors were tilted in the same direction. The shortest intermolecular distance was $3.511 \AA$. The tilt angle of the phthalocyanine core normal vectors from the $a$ axis was $67.9^{\circ}$. Significant changes in the structure of the bulk crystal 
due to the crystal phase transition could not be observed.

On the other hand, the needle crystal structure at $93 \mathrm{~K}$ was determined to be the monoclinic structure with space group $P 2_{1} / n, a=20.96150(10) \AA, b=9.54830(10) \AA, c$ $=35.1736(3) \AA, \alpha=90^{\circ}, \beta=94.0473(3)^{\circ}, \gamma=90^{\circ}$, and $V=7022.32 \AA^{3}$. The unit cell contained four molecules and the density was $1.122 \mathrm{~g} / \mathrm{cm}^{3}$. The column axis was parallel to the $b$ axis and the lattice constant $b$ was the nearly twice the distance of the stack interval. It was observed that the direction of the phthalocyanine core normal vectors alternates from column to column to give a herringbone-like packing structure. The shortest intermolecular distance was $3.554 \AA$ and the tilt angle of the phthalocyanine core normal vectors from the $b$ axis was $40.79^{\circ}$. The needle crystal structure at $93 \mathrm{~K}$ and that in the spin-coated film are considered to be almost the same. Figure 4 shows the molecular packing structure of the needle crystal in the direction of the columnar axis and the in-plane direction at $93 \mathrm{~K}$.

The structure of the needle crystal was also investigated at a higher temperature than the crystal phase transition temperature. The needle crystal structure at $273 \mathrm{~K}$ was determined to be the monoclinic structure with space group $P 2_{1} / n, a=20.7430 \AA, b=$ $9.7984 \AA, c=36.0961 \AA, \alpha=90^{\circ}, \beta=93.8750^{\circ}, \gamma=90^{\circ}$, and $V=7319.7 \AA^{3}$. The unit cell contained four molecules and the density was $1.077 \mathrm{~g} / \mathrm{cm}^{3}$. The shortest intermolecular distance was $3.644 \AA$ and the tilt angle of the phthalocyanine core normal vectors from the $b$ axis was $40.78^{\circ}$. There was no marked change in the structure of the needle crystal due to the crystal phase transition, and the lattice parameters in the needle crystal almost coincided with those in the spin-coated films. Table 1 summarizes the lattice parameters in the bulk and needle crystals at $273 \mathrm{~K}$ and in spin-coated films at room temperature. 
Figure 5 shows the temperature dependences of the lattice parameters in the needle crystal, which confirmed a volumetric change. The lattice parameters in the needle crystal at $123 \mathrm{~K}$ were $a=21.024 \AA, b=9.543 \AA$, and $c=35.260 \AA$. With increasing the temperature, $a$ decreased, while $b$ and $c$ gradually increased. The lattice parameters in the needle crystal at $293 \mathrm{~K}$ were $a=20.652 \AA, b=9.875 \AA$, and $c=36.232 \AA$. The volume of the unit cell monotonically increased and the density decreased as the temperature increased. It is clear that the lattice constants in the needle crystal approached those in the spin-coated film as the temperature increased. The slight difference in the lattice parameters between the needle crystal and spin-coated films was considered to be due to caused by the measurement process, such as the conditions of the temperature change. From these results, it is considered that the structure of $\mathrm{C}_{6} \mathrm{PcH}_{2}$ in the spin-coated films can be identified as needle crystals. Therefore, the molecular packing structure in the spin-coated films was determined to be the herringbone-like packing structure as shown in Fig. 1(c).

The mechanism of the selective crystal growth was investigated by DSC and XRD analysis. Figure 6 shows the powder XRD patterns of the bulk crystal on heating from a room temperature to $373 \mathrm{~K}$ with a heating rate of $5^{\circ} \mathrm{C} / \mathrm{min}$ and rapid cooling to a room temperature. The XRD peak of $2 \theta=4.42^{\circ}(20.0 \AA)$ at a room temperature was generally consistent with the plane distance (001) of the bulk crystal at $273 \mathrm{~K}(19.7 \AA)$. After heating at $373 \mathrm{~K}$, the XRD peak shifted at $2 \theta=4.79^{\circ}(18.4 \AA)$ and a weak peak appeared at $2 \theta=5.07^{\circ}(17.4 \AA)$. The peaks were corresponded with the plane distance (002) (18.0 $\AA$ ) and (101) (17.5 ̊) of the needle crystal at $273 \mathrm{~K}$. The phase transition from the bulk crystal to the needle crystal was also validated by DSC. After cooling to room 
temperature, the XRD peak maintained at $2 \theta=4.83^{\circ}(18.3 \AA)$. The result indicates the irreversibility of the temperature induced transition from the bulk to needle crystals.

The position of the alkyl substituents of the bulk crystal was better order than that of the needle crystal. Since the alkyl substituents move actively at a high temperature, the order of the alkyl substituents were disturbed, resulting in the transform from the bulk crystal to the needle crystal. Therefore, it is considered that the crystal growth condition at a low temperature must be necessary for fabrication of the bulk crystal.

In case of the spin-coating film of $\mathrm{C} \mathrm{PcH}_{2}$, the film was rapidly formed before the alkyl substituents are stabilized to the most stable state, like the bulk crystal, therefore, the needle crystal-like structure was selectively formed in the spin-coated films.

\section{CONCLUSION}

The structures of single-crystal $\mathrm{C}_{6} \mathrm{PcH}_{2}$ were investigated by single-crystal X-ray structure analysis. Two types of $\mathrm{C}^{6} \mathrm{PcH}_{2}$ single crystal, bulk and needle crystals, were grown separately. By the thermal behavior, the bulk crystals were fabricated with priority at a lower temperature than the needle crystals. By the crystal structure analysis, the bulk and needle crystals were determined to be triclinic with space group $P-1$ and monoclinic with space group $P 2_{1} / n$, respectively. It was found that $\mathrm{C}_{6} \mathrm{PcH}_{2}$ exhibits structural polymorphism. According to the observed temperature dependences in the crystal phase, bulk and needle crystals maintain their structures while undergoing

volumetric changes with increasing temperature. Since the structure of $\mathrm{C} \mathrm{PcH}_{2}$ microcrystals in spin-coated films coincided with that of needle crystals at around room temperature, it is considered that in the spin-coating process, $\mathrm{C}_{6} \mathrm{PcH}_{2}$ tends to selectively form the same crystal structure as that of the needle crystals. 


\section{Acknowledgements}

We thank Dr. Yo Shimizu and Dr. Makoto Yoneya of National Institute of Advanced Industrial Science and Technology for helpful suggestions about the molecular packing structure of $\mathrm{C} \mathrm{PcH}_{2}$. This work was partially supported by the Advanced Low Carbon Technology Research and Development Program from the Japan Science and Technology Agency and the JSPS KAKENHI Grant Numbers 15H03552, 24246009, 26600073 and a Grant-in-Aid for JSPS Fellows (No.15J00448) from the Japan Society for the Promotion of Science, and Photonics Advanced Research Center at Osaka University. 


\section{References}

1. R. Zeis, T. Siegrist, C. Kloc, Single-crystal field-effect transistors based on copper phthalocyanine, Appl. Phys. Lett. 86 (2005) 10-13. doi:10.1063/1.1849438.

2. C.W. Tang, Two-layer organic photovoltaic cell, Appl. Phys. Lett. 48 (1986) 183. doi:10.1063/1.96937.

3. K. Sakai, and M. Hiramoto, Efficient Organic p-i-n Solar Cells Having Very Thick Codeposited i-Layer Consisting of Highly Purified Organic Semiconductors, Mol. Cryst. Liq. Cryst., 491 (2008) 284-289. doi:10.1080/15421400802330960.

4. H. Iino, J. Hanna, R.J. Bushby, B. Movaghar, B.J. Whitaker, M.J. Cook, Very high time-of-flight mobility in the columnar phases of a discotic liquid crystal, Appl. Phys. Lett. 87 (2005) 132102. doi:10.1063/1.2056608.

5. H. Iino, Y. Takayashiki, J.I. Hanna, R.J. Bushby, Fast ambipolar carrier transport and easy homeotropic alignment in a metal-free phthalocyanine derivative, Japanese J. Appl. Physics, Part 2 Lett. 44 (2005) 41-44. doi:10.1143/JJAP.44.L1310.

6. T. Hori, Y. Miyake, T. Masuda, T. Hayashi, K. Fukumura, H. Yoshida, et al., Dependence of alkyl-substituent length for bulk heterojunction solar cells utilizing 1,4,8,11,15,18,22,25-octaalkylphthalocyanine, J. Photonics Energy. 2 (2012) 021004. doi:10.1117/1.JPE.2.021004.

7. Q.D. Dao, T. Saito, S. Nakano, H. Fukui, T. Kamikado, A. Fujii, et al., Alkyl substituent length dependence of octaalkylphthalocyanine bulk heterojunction solar cells, Appl. Phys. Express. 6 (2013). doi:10.7567/APEX.6.122301.

8. J.C. Swarts, E.H.G. Langner, N. Krokeide-Hove, M.J. Cook, Synthesis and electrochemical characterisation of some long chain 
$1,4,8,11,15,18,22,25$-octa-alkylated metal-free and zinc phthalocyanines possessing discotic liquid crystalline properties, J. Mater. Chem. 11 (2001) 434-443. doi:10.1039/b006123i.

9. A.S. Cherodian, A.N. Davies, R.M. Richardson, M.J. Cook, N.B. Mckeown, A.J. Thomson et al., Mesogenic Behaviour of some 1,4,8,11,15,18,22,25-Octa-alkylphthalocyanines, Mol. Cryst. Liq. Cryst., 196 (1991) 103-114. doi: 10.1080/00268949108029690.

10. Y. Miyake, Y. Shiraiwa, K. Okada, H. Monobe, T. Hori, N. Yamasaki, et al., High Carrier Mobility up to $1.4 \mathrm{~cm}^{2} \cdot \mathrm{V}^{-1} \cdot \mathrm{s}^{-1}$ in Non-Peripheral Octahexyl Phthalocyanine, Appl. Phys. Express. 4 (2011) 021604. doi:10.1143/APEX.4.021604.

11. T. Hori, Y. Miyake, N. Yamasaki, H. Yoshida, A. Fujii, Y. Shimizu, et al., Solution processable organic solar cell based on bulk heterojunction utilizing phthalocyanine derivative, Appl. Phys. Express. 3 (2010) 2-4. doi:10.1143/APEX.3.101602.

12. M. Ohmori, H. Fukui, Q.D. Dao, T. Kumada, A. Fujii, Y. Shimizu, et al., Annealing effect in bulk heterojunction organic solar cells utilizing liquid crystalline phthalocyanine, Jpn. J. Appl. Phys. 53 (2014) 1-4. doi:10.7567.

13. Q.D. Dao, T. Hori, K. Fukumura, T. Masuda, T. Kamikado, A. Fujii, et al., Effects of processing additives on nanoscale phase separation, crystallization and photovoltaic performance of solar cells based on mesogenic phthalocyanine, Org. Electron. 14 (2013) 2628-2634. doi:10.1016/j.orgel.2013.05.041.

14. C. Piechocki, J. Simon, A. Skoulios, D. Guillon, P. Weber, Annelides. 7. Discotic mesophases obtained from substituted metallophthalocyanines. Toward liquid crystalline one-dimensional conductors, J. Am. Chem. Soc., 104 (1982) 5245-5247. doi:10.1021/ja00383a050. 
15. B.J. Simmerer, B. Gliisen, W. Paulus, A. Kettner, P. Schuhmacher, D. Adam, et al., Transient Photoconductivity in a Discotic Hexagonal Plastic Crystal, Adv. Mater. 8(10) (1996) 815-819. doi:10.1002/adma.19960081010.

16. Y. Shimizu, Y. Miyake, H. Yoshida, H. Monobe, M.J. Cook, A. Fujii, et al., A Possibility of 2-Dimensional Transport of Charged Carriers in Columnar Phases of Liquid Crystalline Semiconductors, Mol. Cryst. Liq. Cryst. 549 (2011) 127-132. doi:10.1080/15421406.2011.581162.

17. M. Yoneya, T. Makabe, A. Miyamoto, Y. Shimizu, Y. Miyake, H. Yoshida, et al., Tilt orientationally disordered hexagonal columnar phase of phthalocyanine discotic liquid crystals, Phys. Rev. E. 89 (2014) 062505. doi:10.1103/PhysRevE.89.062505.

18. M. Ohmori, T. Higashi, A. Fujii, M. Ozaki, Molecular Packing Structure of Mesogenic Octa-Hexyl Substituted Phthalocyanine Thin Film by X-ray Diffraction Analysis, 16 (2016) 3318-3321. doi:10.1166/jnn.2016.12288.

19. I. Chambrier, M.J. Cook, A.K. Powella, X-Ray Crystal Structure of a Mesogenic Octa-substituted Phthalocyanine, J. Chem. Soc. Chem. Commun. (1992) 444-445.

20. J. Tant, Y.H. Geerts, M. Lehmann, V. De Cupere, G. Zucchi, B.W. Laursen, et al., Liquid crystalline metal-free phthalocyanines designed for charge and exciton transport, J. Phys. Chem. B. 109 (2005) 20315-20323. doi:10.1021/jp054778o.

21. M. Yoneya, A. Miyamoto, Y. Shimizu, A. Fujii, M. Ozaki, Origin of the High Carrier Mobilities of Nonperipheral Octahexyl Substituted Phthalocyanine, J. Phys. Chem. C. 119 (2015) 23852-23858. doi:10.1021/acs.jpcc.5b07085. 


\section{Figure Caption}

Figure 1. Schematic explanation of the molecular alignment in the spin-coated film of $\mathrm{C}_{6 \mathrm{PcH}}$ from the direction of columnar axis (a) and in-plane direction (b) (c). Two candidates of the molecular packing structures, in which the molecules tilt uniformly (b) or alternately (c), are considered.

Figure 2. Microscope images of the bulk (a) and needle (b) crystals of $\mathrm{C}_{6} \mathrm{PcH}_{2}$.

Figure 3. Molecular packing structure of the bulk crystal of $\mathrm{C}_{6} \mathrm{PcH}_{2}$ in the direction of columnar axis (a) and in-plane direction (b) at $213 \mathrm{~K}$. The red, green, blue lines indicates $a, b$, and $c$ axes, respectively.

Figure 4. Molecular packing structure of the needle crystal of $\mathrm{C} \mathrm{PcH}_{2}$ in the direction of columnar axis (a) and in-plane direction (b) at $93 \mathrm{~K}$. The red, green, blue lines indicates $a, b$, and $c$ axes, respectively.

Figure 5. Temperature dependence of lattice parameters of $a, b$, and $c$ in the needle crystal of $\mathrm{C}_{6} \mathrm{PcH}_{2}$.

Figure 6. Changes of the powder XRD patterns of the bulk crystal with heating from a room temperature to $373 \mathrm{~K}$ and cooling to a room temperature. 
Table 1. The lattice parameters in the bulk and needle crystals at $273 \mathrm{~K}$ and the molecular packing structure in spin-coated films at room temperature.

\begin{tabular}{|c||c|c|c|c|c|c|}
\hline \multicolumn{1}{|c||}{$\begin{array}{c}\text { Crystalline state } \\
\text { of } \mathrm{C}_{\mathrm{PcH}}\end{array}$} & $a$ & $b$ & $c$ & $\alpha$ & $\beta$ & $\gamma$ \\
\cline { 2 - 7 } & $9.31 \AA$ & $9.79 \AA$ & $19.98 \AA$ & $100.5^{\circ}$ & $91.1^{\circ}$ & $95.6^{\circ}$ \\
\hline \hline Bulk crystal & $20.74 \AA$ & $9.80 \AA$ & $36.10 \AA$ & $90^{\circ}$ & $93.9^{\circ}$ & $90^{\circ}$ \\
\hline Needle crystal & Lattice parameters \\
\hline Spin-coated film & $20.3 \AA$ & $4.8-5.0 \AA$ & $36.4 \AA$ & $90^{\circ}$ & $90^{\circ}$ & $90^{\circ}$ \\
\hline
\end{tabular}




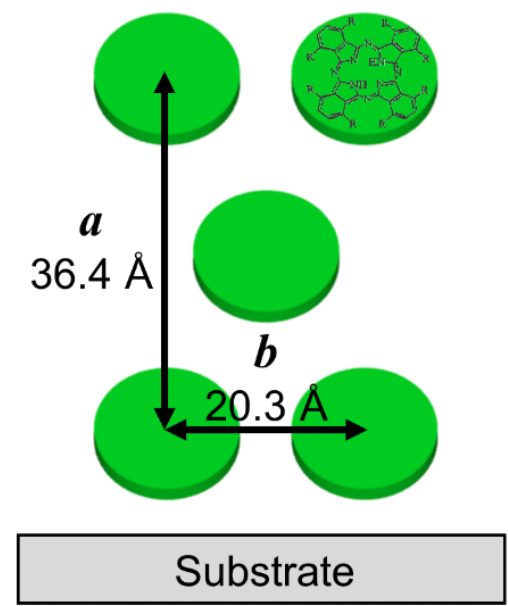

(a)

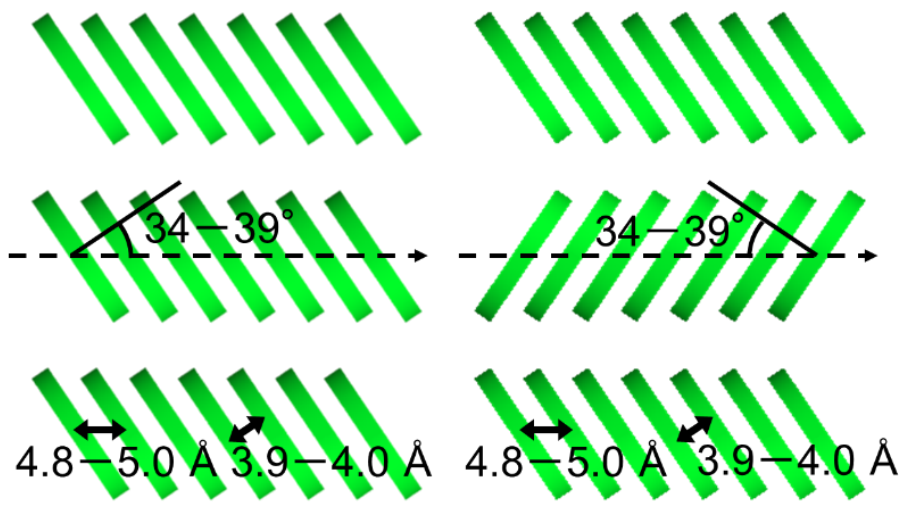

Substrate

(b)

\section{Substrate}

Figure 1. M. Ohmori et al. 

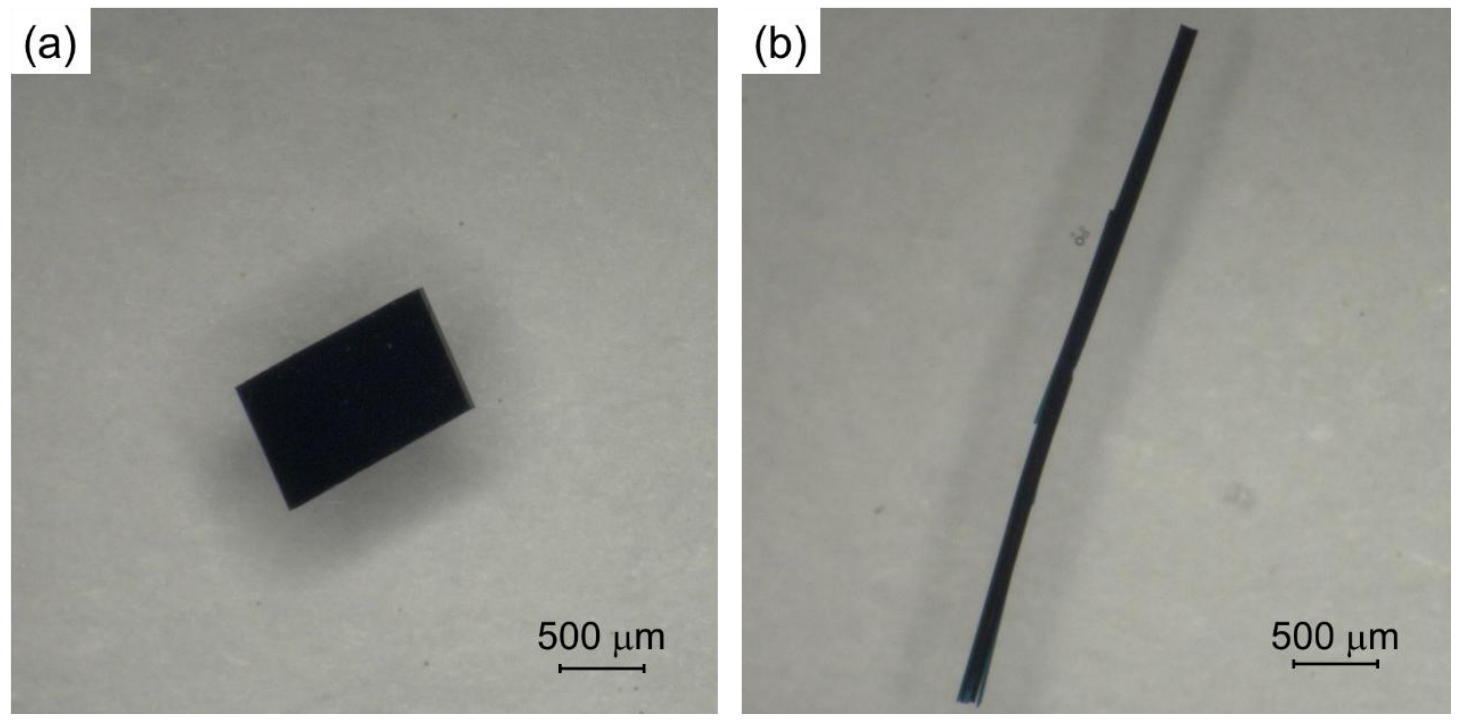

Figure 2. M. Ohmori et al. 


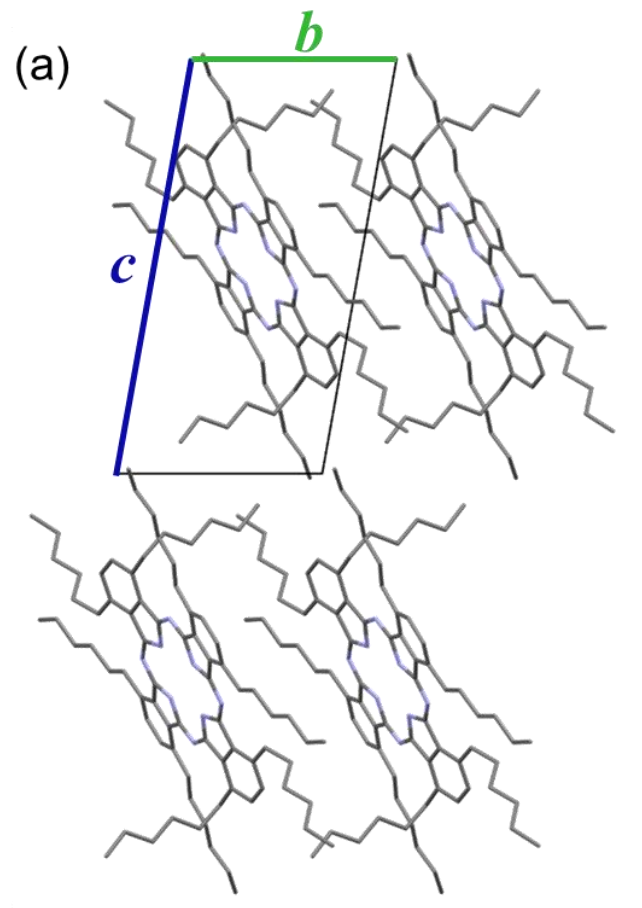

(b)

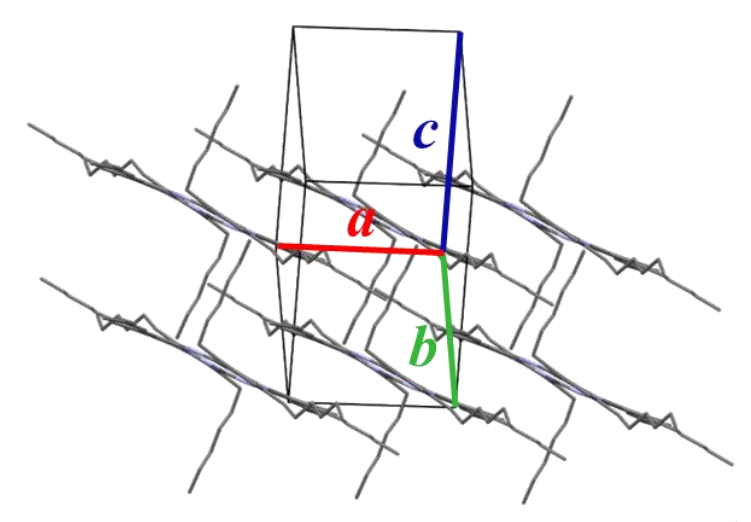

Figure 3. M. Ohmori et al. 


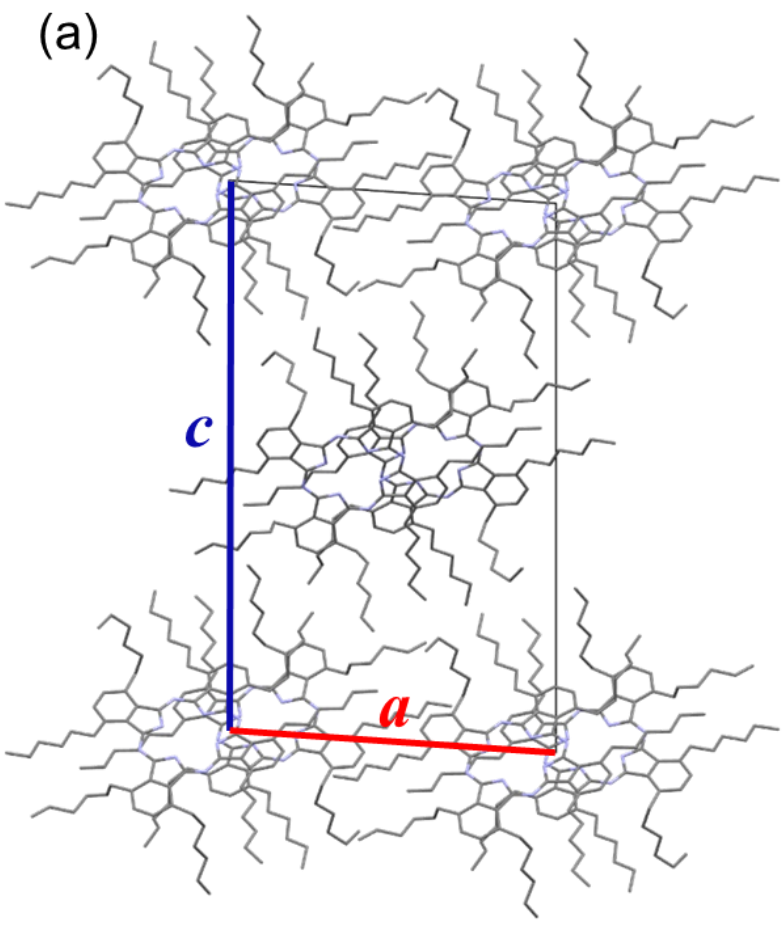

(b)

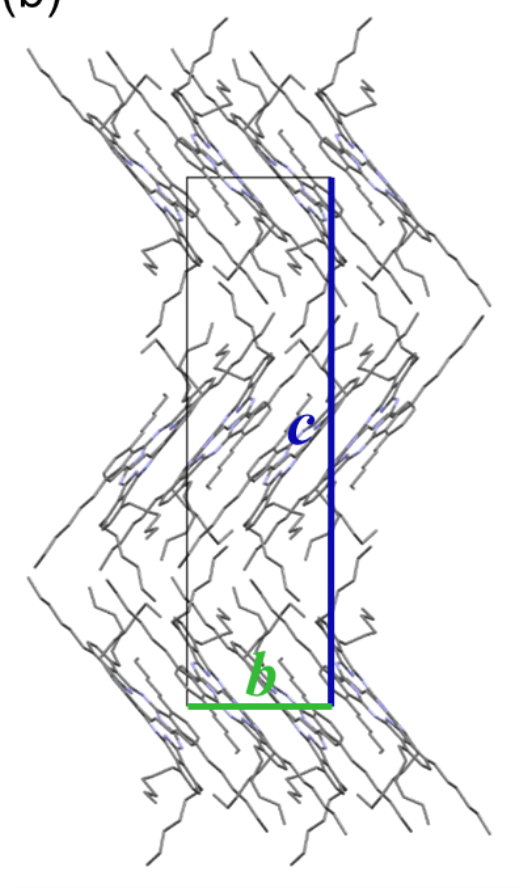

Figure 4. M. Ohmori et al. 

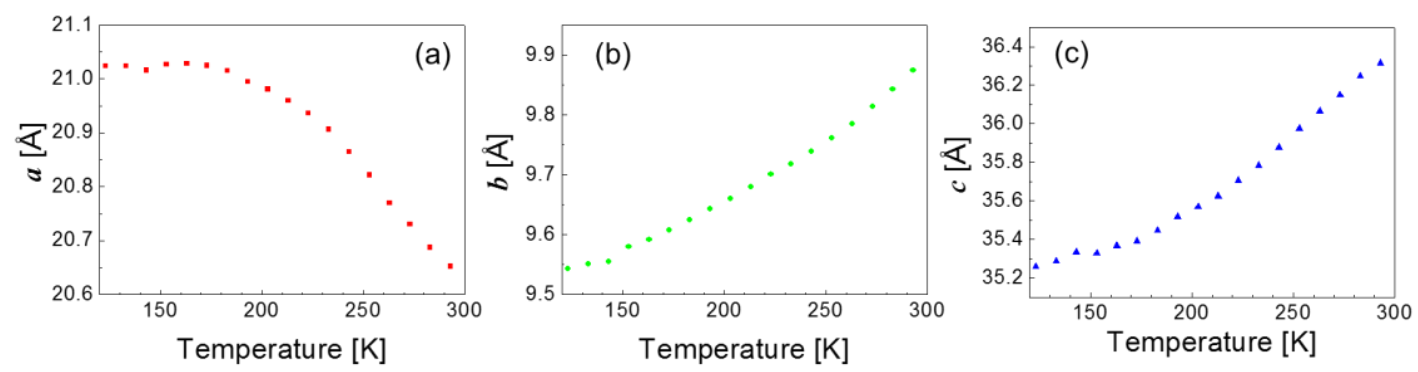

Figure 5. M. Ohmori et al. 


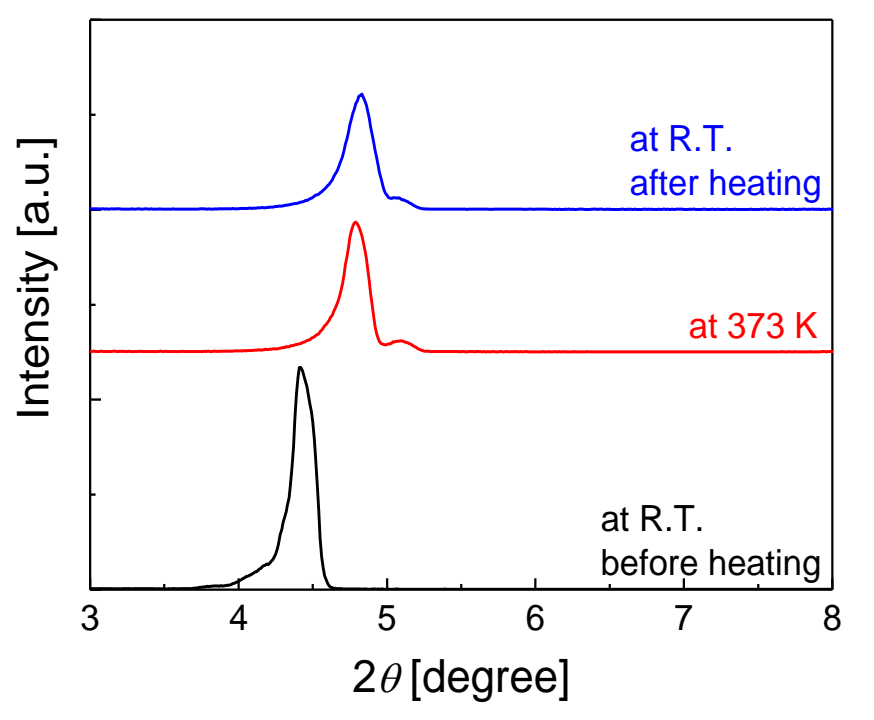

Figure 6. M. Ohmori et al. 


\section{Graphical abstract}

Two types of non-peripheral octahexyl-substituted phthalocyanine single-crystal structures have been investigated in order to clarify the structure in thin films used in electronic devices.

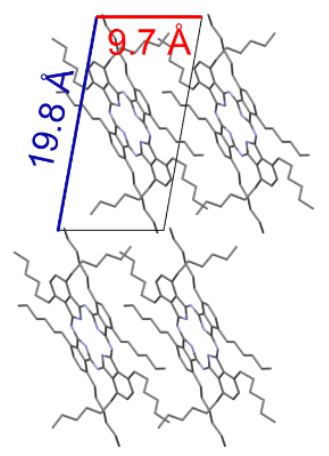

Bulk Crystal

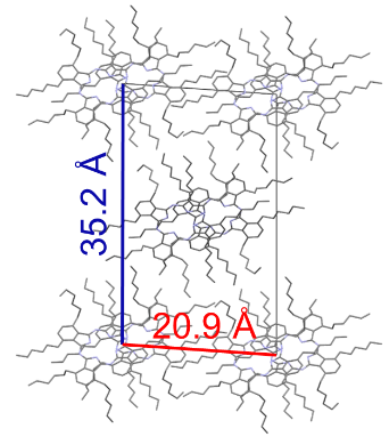

Needle Crystal

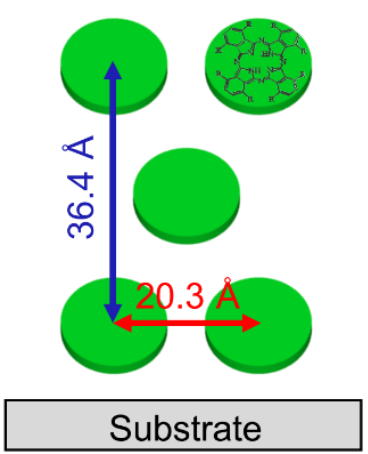

Thin Film 